\title{
Modeling and Simulation of Adaptive Surface Tracked Vehicle Based on RecurDyn
}

\author{
Wei Chen, Jianming Zhan, Hang Zhou \\ Faculty of Mechanical Engineering and Mechanics, Ningbo University, Ningbo, China \\ Email: flynchen@aliyun.com
}

Received July 3, 2013; revised August 5, 2013; accepted August 20, 2013

Copyright (C) 2013 Wei Chen et al. This is an open access article distributed under the Creative Commons Attribution License, which permits unrestricted use, distribution, and reproduction in any medium, provided the original work is properly cited.

\begin{abstract}
In order to improve the adaptability of the tracked vehicle in the road and strengthen the grip of the tracked vehicle, a track surface adaptive mechanism was provided. In theory, it has been proved practically. Meanwhile, RecurDyn, which is a multi-body kinematics software, was used to build a multi-body soft hybrid model, based on structure, elasticity, linear damping adaptive tracked vehicle; meanwhile the model was used to carry on the kinematics simulation. Through the comparison between simulated motion trail and that of traditional motion trail, this paper analyzed the deviation of the motion trail and also simulated the motion trail of the warped surface so as to test the adaptive ability of the mechanism. According to the results, the adaptive mechanism was equipped with great surface adaptability. It can also adapt to the complex warped surface, and enjoy a damping effect.
\end{abstract}

Keywords: Warped Surface Adaptation; Tracked Vehicle; Modeling and Simulation; Adaptive Mechanism

\section{Introduction}

Due to the great load ability, big traction and the small unit area pressure, the tracked vehicle is always regarded as the army's important combat equipment. With the needs of heavy-motorized modern warfare, tracked vehicle must be adapted to a variety of complex and harsh terrain to have a stronger ability to survive in future wars [1]. However, because the traditional model of tracked vehicle was based on empirical formula and a large number of tests with lengthy development cycle, tracked vehicle developed very slowly. Especially when driving high-speed in the irregular terrain, the running gear of the tracked vehicle is likely to fracture due to the uneven fore of the track. When the vehicle turns, due to the lack of grip, it is likely to cause skidding, even in the side turn $[2,3]$. In this paper, combined with the practical issues of a surface adaptive mechanism for tracked vehicle, based on virtual manufacturing technology, the $3 \mathrm{D}$ simulation model of the tracked vehicle is established through the multi-body software RecurDyn to simulate its adaptive ability [4].

\section{Adaptive Mechanism Principle Analysis and Prototype Building}

\subsection{Adaptive Mechanism Principle Analysis}

Based on the practical problem mentioned above, it is important to improve the grip and good ground adaptability. In many engineering practice, piecewise linear fitting curve is often used to obtain an approximate curve [5]. According to this idea, we can make rational improvement of tracked vehicle suspension system to design a surface adaptive mechanism, as is seen in Figure 1 and to let the crawler stick to the ground all the time, so it can solve the grip and ground adaptability problems mentioned above [6].

The basic principle of adaptive mechanism is to add a certain number of spring dampers in closed areas and to add rollers in the dampers below, so it is different from the traditional rollers of tracked vehicle. The roller inter-functioning with spring dampers can be posted on the track, so that it can move in perpendicular direction as

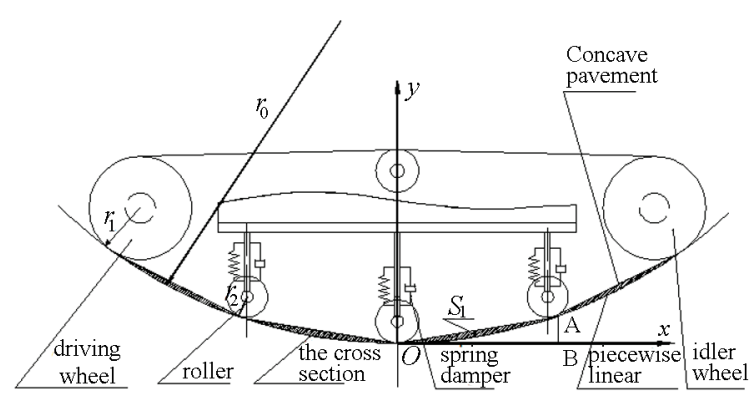

Figure 1. Adaptive mechanism principle diagram. 
well as roll in the inner wall of the track at the same time, so the caterpillar chain can always stick to the ground to run, improving the safety performance of tracked vehicles.

According to the actual analysis, the smaller the gap between the track and concave surface contact is, the better the pavement sticking effect can be. So the cross section area between the track and the concave surface needs to be solved to know its adaptive effect. Suppose the caterpillar chain is rigid, and the inducer under the effect of the dampers in the movement process causes little change of the track, so it can be put aside. That is to say, the caterpillar chain length on the ground will not change. Suppose the adaptive surface length is $l_{0}, \mathrm{n}-1$ spring dampers are installed between the driving wheel and the idler wheel and the adaptive track on the ground is divided into $\mathrm{n}$ equally without considering the spring damper size and installation dimensions, so each length is $l_{0} / n$, as is shown in Figure 1, $O A=l_{0} / n$. Therefore, the gap area of track and the concave surface is the same. As the coordinate system established in the Figure 1 shows, suppose the area between the two adjacent spring damping areas is $S_{i}(i=1,2,3, \cdots, n)$, so the gap area of the track and the concave surface is

$$
\begin{aligned}
S=\sum_{i=1}^{n} S_{i}=S_{1}+S_{2}+\ldots S_{n} & \quad \text { so when Equations (1)-(5) is com } \\
\lim _{n \rightarrow \infty} S & =\lim _{n \rightarrow \infty} n S_{1}=\lim _{n \rightarrow \infty} n\left(S_{1}^{\prime}-S_{1}^{\prime \prime}\right) \\
& =\lim _{n \rightarrow \infty}\left[\frac{l_{0}^{2} \sin 2 \theta}{4 n^{2}}-r_{0}^{2}\left(\sin 2 \theta-\frac{1}{2} \arcsin \sin 2 \theta-\frac{1}{4} \sin 4 \theta\right)\right] \\
& =\lim _{n \rightarrow \infty}\left[\frac{l_{0}^{2}}{4 n}\left(\sin 2 \theta-2 \cot \theta+\frac{\theta}{\sin ^{2} \theta}+\cot \theta \cos 2 \theta\right)\right] \\
& =\lim _{n \rightarrow \infty}\left[\frac{l_{0}}{4} \times \frac{l_{0}}{n}\left(\sin 2 \theta+(\cos 2 \theta-2 \cot \theta)+\frac{\theta}{\sin ^{2} \theta}\right)\right]
\end{aligned}
$$

Because of the equal distance, the overall gap area is $S=\sum_{i=1}^{n} S_{i}=n S_{1}$

The area of $S_{1}$ is $S_{1}^{\prime}$ of $\triangle O A B$ minus $S_{1}^{\prime \prime}$ of $O \hat{A} B$, that is $S_{1}=S_{1}^{\prime}-S_{1}^{\prime \prime}$.

Suppose $\angle \mathrm{AOB}=\theta, \quad \theta \in\left(0, \frac{\pi}{2}\right)$;

It can be inferred that

$$
\begin{gathered}
S_{1}^{\prime \prime}=\int_{0}^{r_{0} \sin 2 \theta}\left(r_{0}-\sqrt{r_{0}^{2}-x^{2}}\right) \mathrm{d} x \\
=\left|r_{0} x-\frac{1}{2} r_{0}^{2} \operatorname{arc} \sin \frac{x}{r_{0}}-\frac{x \sqrt{r_{0}^{2}-x^{2}}}{2}\right|_{0}^{r_{0} \sin 2 \theta}
\end{gathered}
$$

Then,

$$
S_{1}^{\prime \prime}=r_{0}^{2}\left(\sin 2 \theta-\frac{1}{2} \arcsin \sin 2 \theta-\frac{1}{4} \sin 4 \theta\right)
$$

And

$$
\begin{gathered}
2 r_{0} \sin \theta=\frac{l_{0}}{n} \\
\frac{l_{0}}{n}=\frac{2 \theta \pi r_{0}}{180^{\circ}}, \text { so } \theta=\frac{90^{\circ} l_{0}}{n \pi r_{0}}
\end{gathered}
$$

so when Equations (1)-(5) is combined, it can be seen: when $n \rightarrow \infty, \theta \rightarrow 0$, so

$$
\begin{aligned}
& =\lim _{n \rightarrow \infty} \frac{l_{0}}{4} \times \frac{l_{0}}{n}\left[\sin 2 \theta+(\cos 2 \theta-2) \cot \theta+\frac{\theta}{\sin ^{2} \theta}\right] \\
& =\lim _{\theta \rightarrow 0} \frac{l_{0}}{4} \times 2 r_{0} \sin \theta\left[\sin 2 \theta+(\cos 2 \theta-2) \cot \theta+\frac{\theta}{\sin ^{2} \theta}\right] \\
& =\lim _{\theta \rightarrow 0} \frac{l_{0} r_{0}}{2} \times\left[\cos \theta\left(2 \sin ^{2} \theta+\cos 2 \theta-2\right)+\frac{\theta}{\sin \theta}\right] \\
& =\frac{l_{0} r_{0}}{2} \lim _{\theta \rightarrow 0}\left(\frac{\theta}{\sin \theta}-\cos \theta\right)=0
\end{aligned}
$$

So when the number of spring dampers as $n$ which tends to infinity, the section area of the track and theconcave surface gap approaches to zero, it is completely attached on the concave surface. But in the actual situation, the number of spring dampers can not be infinite, but spring damper of tracked vehicle of a certain number helps track attached on the road. In this paper, the tracked vehicle with two groups of spring dampers, each with 3 is symmetrically installed in the track inside a closed interval to establish an adaptive model of tracked vehicles.

\subsection{The Establishment of 3D Simulation Prototype}

Multi-body dynamics software RecurDyn is a new generation of multi-body dynamics simulation software, especially with outstanding performance in the solution of 
large-scale multi-body contact problems. RecurDyn provides a rich toolkit application subsystem with the characteristic of a fast and convenient modeling and solving process on the bases of parameterization, modular modeling technology and professional knowledge and experience. In this paper, the toolkit is track package. The various components of the tracked vehicle are defined as a rigid body or flexible body through various constraints in combination to quickly establish a simulation of tracked vehicle model and various forms of kinematics simulation $[7,8]$.

Specific operation method is: on the basis of the relevant dimension position and relationship, establishing the three-dimensional model of the main components in RecurDyn to get the geometric size of the simulation model, the installation position and the performance parameters. At last, the module of the track package is used to assemble to get the adaptive tracked vehicle simulation model of 1:1 3D model, as shown in Figure 2. Then in order to be sure of the motion constraint of simulation movement, spring damper constraint between the roller and the track is established, suppose it is linear.

\section{Adaptive Kinematics Simulation and Analysis of Tracked Vehicle}

\subsection{Adaptive Analysis of Traditional Tracked Vehicle and Adaptive Vehicle}

Based on the above, the smaller gap of the track and the road cross-sectional area is, the better capacity of grip the road for tracked vehicle has, and further shows that when trajectory error between the track and the road is smaller, surface adaptive is better. Two kinds of vehicles in this paper refer to the tracked vehicle in the traditional sense and a self-adaptive mechanism respectively. First is to establish the ground spline curve in RecurDyn. This paper mainly selects two basic function $\mathrm{Y}=\mathrm{k}$ ( $\mathrm{k}$ constant) and $Y=A \sin (\omega t+\psi)$ to simulate the flat pavement and uneven surface pavement and use the STEP function as driving conditions of the tracked vehicle, where $0-0.4 \mathrm{~s}$

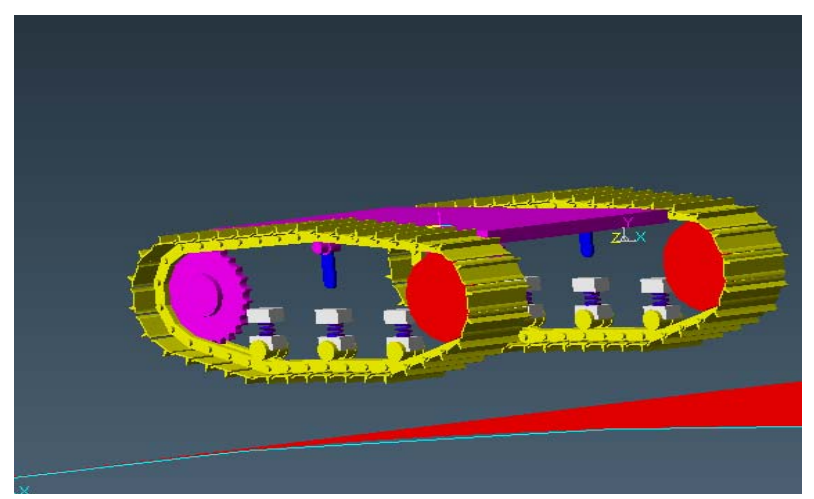

Figure 2. Adaptive tracked vehicle simulation model. denotes free fall on the road of the tracked vehicle, 0.4 $2 \mathrm{~s}$ simulation of flat pavement, $2-10 \mathrm{~s}$ simulation on uneven surface pavement conditions and the same road conditions were built in RecurDyn. In Figure 3, the two solid lines represent the comparison of two kinds of vehicle wheel motion amplitude, while the dotted line represents the pavement track of the tracked vehicle.

Suppose a single point of the pavement is $|\sigma|$, there are:

$$
\begin{aligned}
& |\sigma|=|t|+l_{1}+l_{2}+\delta \\
& c=l_{1}+l_{2}
\end{aligned}
$$

$\mathrm{T}$ - Wheel centroid coordinates

$l_{1}$-Track thickness

$l_{2}$ - Roller centroid height

$\delta$ - The trajectory error

If we know the track thickness and height of centroid track roller, so trajectory error of the tracked vehicle at a point is: $\delta=|\sigma|-|t|-c$

The adaptive tracked vehicle is set $\mathrm{c}=140 \mathrm{~mm}$.

According to the Figure 3, maximum point Max of the trajectory error is in the peaks and troughs. The maximum error can be calculated according to the above formula and the specific trajectory error can be seen in Table 1 .

From Table 1, experimental data shows that the trajectory error of the adaptive tracked vehicle is significantly less than the traditional tracked vehicle. The two tracked vehicle trajectory error ratio can also reflect this problem, especially in the trough. The minimum ratio trajectory error of the traditional tracked vehicle indicates the poor adaptive effect particularly. From the Figure 3, it can be seen when the free fall of the tracked vehicle walks on flat pavement, the traditional tracked vehicle has obvious concussion. Such shocks are very detrimental to the stability of tracked vehicles while the adaptive vehicle is a smooth curve; When the tracked vehicle walks along the rugged road, in the uphill process two amplitude curves coincide, but in the low slope, the fall

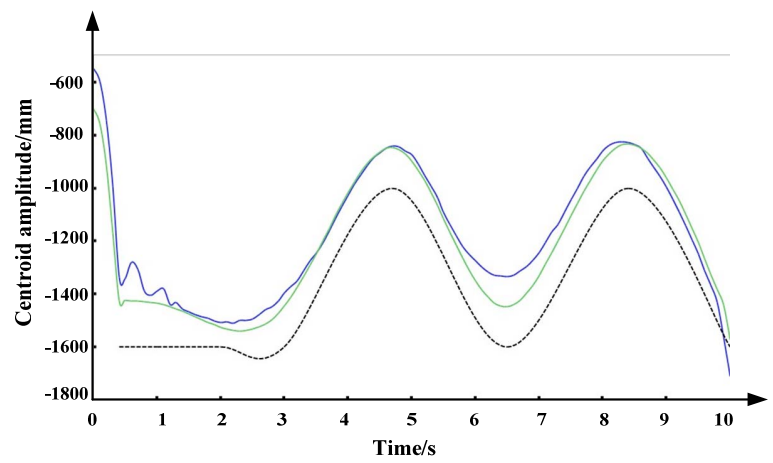

Figure 3. Middle wheel centroid amplitude comparison of two kinds of vehicles. 
Table 1. The maximum tracking error of the tracked vehicles running on the road.

\begin{tabular}{cccc}
\hline & The first peak & The first trough & The second peak \\
\hline Coordinates Of peak or trough $|\sigma| / \mathrm{mm}$ & -1000 & -1600 & -1000 \\
the adaptive track vehicle $|t| / \mathrm{mm}$ & -847 & -1448 & -837 \\
traditional tracked vehicle $|t| / \mathrm{mm}$ & -831 & -1310 & 12 \\
Trajectory error $\quad \delta_{1} / \mathrm{mm}$ & 13 & 150 & -824 \\
Trajectory error $\quad \delta_{2} / \mathrm{mm}$ & 29 & $8 \%$ & 36 \\
Trajectory error ratio $\quad \delta_{1} / \delta_{2}$ & $44.8 \%$ & & $63.9 \%$
\end{tabular}

of the adaptive vehicle is more obvious, which shows that the adaptive mechanism is good to the damping effect of the tracked vehicle, directly attached to the concave wall, and can guarantee the tracked vehicle is very safe when driving. Figure 4 shows the acceleration the two kinds of tracked vehicle body centroid changes in 10s. From the figure we can see that the acceleration of the traditional tracked vehicle centroid fluctuates greatly, especially in the position of peaks and troughs, and adaptive tracked vehicle only fluctuate in a certain range, which shows adaptive mechanism greatly reduces running impact load of the tracked vehicle on uneven road, and ensures the grip of tracked vehicle in the driving process. According to the simulation results, the greater the slope is, the more obvious the gap of the amplitude fluctuation between adaptive tracked vehicles and the traditional tracked vehicle is.

\subsection{Analysis of Road Adaptive Ability}

The so-called complex road conditions, is that tracked vehicle walks on the warping surface; the pavement is an irregular curved surface .First, building two spline curves of $Y=A \sin (\omega t+\psi)$ and $Y=A \cos (\omega t+\psi)$ in RecurDyn, as is shown in Figure 5 to simulate the moving conditions on the complex road of the adaptive tracked vehicles. The change of the middle wheel centroid amplitude of the self-adaptive tracked vehicle tends to trigonometric function, as is seen in Figure 6, at the same time interval with phase, which shows that the adaptive tracked vehicles is greatly attached to walk on the complex curved surface.

\section{Conclusions}

1) Taking the inextensible cable as the premise, and establishing the specific method, the curved adaptive ability is described. The gap area between the track and the road is to determine the effect of adaptability, which lays a basic foundation for the adaptive description of the tracked vehicle on the complex road.

2) According to the trajectory error, compared with the traditional tracked vehicle, the adaptive mechanism of adaptive tracked vehicles has very strong adaptability,

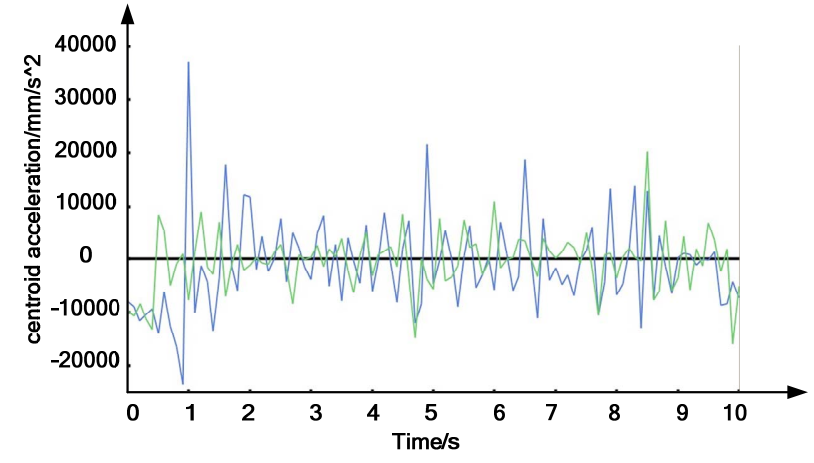

Figure 4. Comparison of two kinds of vehicle body centroid acceleration.

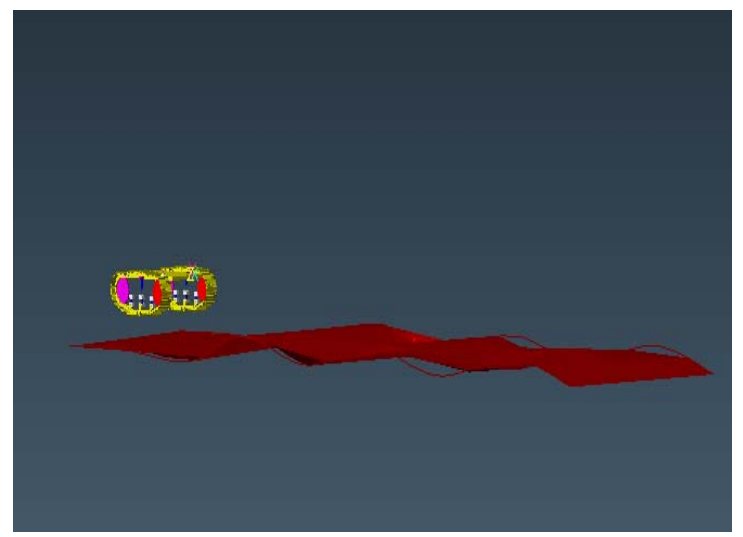

Figure 5. Spline warping pavement.

especially achieves great adaptive effect in the concave surface.

3) Based on the $Y=A \sin (\omega t+\psi)$ spline curve road to simulate the regular surface and curved surface road, ground motion model of the tracked vehicle expanses from $2 \mathrm{D}$ to $3 \mathrm{D}$ space to analyze the asymmetric load situation. It is of strong significance to combine with the reality of tracked vehicle.

\section{Acknowledgements}

All the research works are supported by the Natural Science Foundation of Zhejiang Province of China under granted No. LY12E05007, the Industrial Major (Key)- 


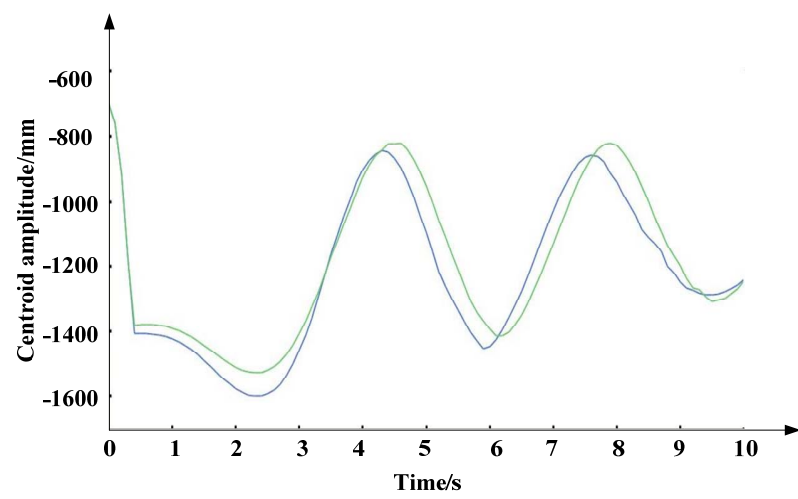

Figure 6. The change of the middle wheel centroid amplitude of the self-adaptive tracked vehicle.

Commissioned Research of Ningbo City of China under granted No. 2012B10057 and the new-shoot Talents Program of Zhejiang province of China under granted No. 2012R405064.

\section{REFERENCES}

[1] Q.-D. Yan, L.-D. Zhang and Y.-Q. Zhao, "Structure and Design of Tank," Institute of Technology Press, Beijing, 2007.

[2] Q.-D. Yan, H.-W. Cui and H.-C. Li, "Study on Steering of Tracked Vehicles on Ramp," Machinery Design \& Manufacture, Vol. 12, 2011, pp. 149-151.

[3] Zhao Yu and H.-W. Yan, "Design Analysis and Research on Tracked Walking Mechanism," New Technology \& New Process, 2010, Vol. 5, pp. 50-53.

[4] B.-K. Han, X.-L. Li and F.-C. Sun, "Present State and Future Outlook of the Simulation of Tracked Vehicles," Acta Armamentar, Vol. 24, No. 2, 2003, pp. 246-249.

[5] E.-L. Li, "A General Theory of the Interpolation Principle in CNC System," National Defence Industrial Press, Beijing, 2008.

[6] J. M. Solis and R. G. Longoria, "Modeling Track-Terrain Interaction for Transient Robotic Vehicle Maneuvers," Journal of Terramechanics, Vol. 45, 2008, pp. 65-78. http://dx.doi.org/10.1016/j.jterra.2008.07.003

[7] X.-J.Jiao, J.-W. Zhang and B.-B. Peng, "Recurdyn MultiBody System Simulation and Optimization Technology," Tsinghua University Press, Beijing, 2010.

[8] D. Rubinstein and R. Hitron, "A Detailed Multi-Body Model for Dynamic Simulation of Off-Road Tracked Vehicles," Journal of Terramechanics, Vol. 41, No. 2-3, 2004, pp. 163-173.

http://dx.doi.org/10.1016/j.jterra.2004.02.004 